NASA TECHNICAL MEMORANDUM NASA TM $X-52115$

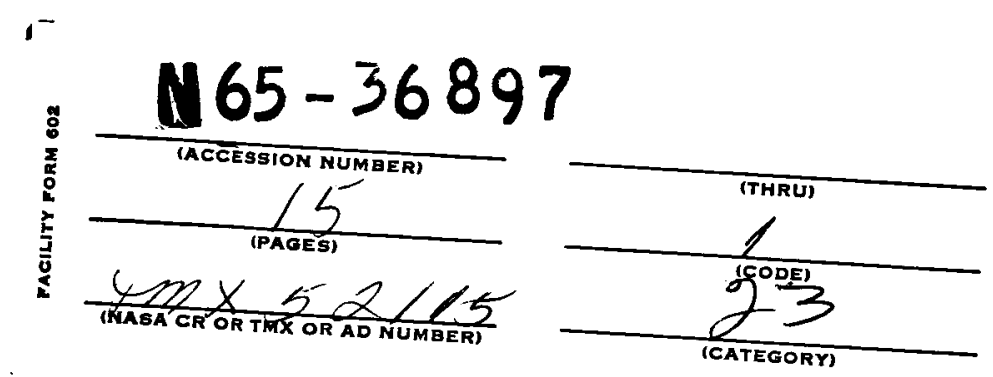

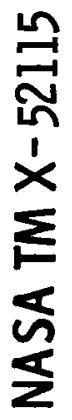

GPO PRICE

$\$$

\section{A SUPERCONDUCTING MAGNETIC BOTTLE}

by James C. Laurence and Willard D. Coles Lewis Research Center Cleveland, Ohio
CFSTI PRICE(S) \$

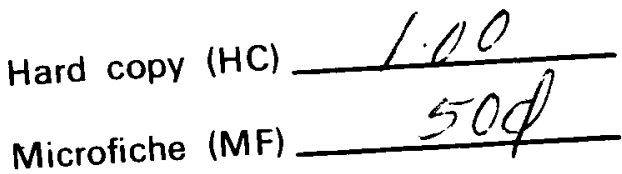

ff 653 July 65

TECHNICAL PREPRINT prepared for Cryogenic Engineering Conference Houston, Texas, August 23-25, 1965. 


\title{
A SUPERCONDUCTING MAGNETIC BOTTLE
}

\author{
by James C. Laurence and Willard D. Coles \\ Lewis Research Center \\ Cleveland, Ohio
}

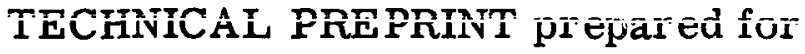

Cryogenic Engineering Conference

Houston, Texas, August 23-25, 1965 


\author{
A SUPERCONDUCTING MAGNETIC BOTTLE \\ by James C. Laurence and Willard D. Coles \\ Lewis Research Center \\ National Aeronautics and Space Administration \\ Cleveland, Ohio \\ ABSTRACT
}

A superconducting magnetic bottle consisting of two mirror coils, a central field coil, and a cylindrical cusp field coil, has been designed and constructed of $\mathrm{Nb}-25 \% \mathrm{Zr}$ cable. The configuration was designed to investigate the problems involved in using superconducting materials to produce magnetic fields useful for experiments in plasma physics. Fields as intense as $50 \mathrm{~kg}$ have been produced in 4-in. inside diameter coils with a superimposed quadrupole cusp field of 5 to 10 percent of the center field and a mirror ratio of 2 .

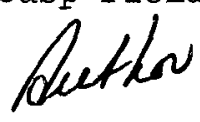

\title{
INTRODUCTION
}

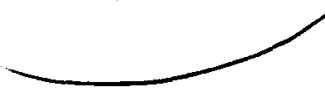

Magnetic fields for plasma physics experiments have been produced in many sizes, configurations, and field strengths. In many cases the experiments have been handicapped by limited power, limited field strength, or duration of experiments by insufficient power or inadequate cooling for the magnet coils. In addition, the heavy coils, support structure, and cooling passages have interfered with free access to the centerline of the magnetic fields.

Now, the rapidly developing techniques of manufacture of superconducting magnets offers a solution to these difficulties while introducing only the additional problem of liquid-helium envirorment for the magnetic coils. The Lewis Research Center of NASA has been studying the application of superconducing magnets to plasma physics. One such apparatus, described by Roth[1], has per- 
formed well in actual use during the past several months. This device consists of a split pair of superconducting coils each enclosed in its separate helium dewar. The separation between the dewars is adjustable by inserting support rods of different lengths. The inner diameter of the $25-\mathrm{kG}$ coils is about 7 in. and the separation can be varied from approximately 6 to 21 in. The system of coils described in this paper was designed to study the use of superconducting coils at higher fields and in other configurations than those now in use for plasma physics experiments at Lewis Research Center. Among the configurations to be studied are those deemed most suitable for stable plasma containment (such as the Ioffe cusp fields ${ }^{[2]}$ ). The mirror coils were designed (using the methods of refs 3 to 5) to produce $50 \mathrm{kG}$ on the central axis (see Fig. 1). A mirror ratio of 2 was provided and a more uniform central field was produced by inserting a third coil between the split pair. In addition a cusp field of 5 to 10 percent of the center field is produced by an array of four linear conductors ("Ioffe bars") equally spaced around the inner diameter of the coil forms. The design, fabrication, and operation of this system of superconducting coils are described.

\section{DESIGN AND FABRICATION OF COILS}

\section{Field Requirements}

The coil design to produce the 2:1 mirror ratio and maximum mirror fields of $50 \mathrm{kG}$ was straightforward. With a minimum inside diameter of $4.0 \mathrm{in}$. and using the minimum wire lengths required to produce the desired fields the coil dimensions and the calculated field were as shown in Table $I$ and Fig. 2. 


\author{
A SUPERCONDUCTING MAGNETIC BOTTLE \\ by James C. Laurence and Willard D. Coles \\ Lewis Research Center \\ National Aeronautics and Space Administration \\ Cleveland, Ohio
}

ABSTRACT

A superconducting magnetic bottle consisting of two mirror coils, a central field coil, and a cylindrical cusp field coil, has been designed and constructed of $\mathrm{Nb}-25 \% \mathrm{Zr}$ cable. The configuration was designed to investigate the problems involved in using superconducting materials to produce magnetic fields useful for experiments in plasma physics. Fields as intense as $50 \mathrm{~kg}$ have been produced in 4-in. inside diameter coils with a superimposed quadrupole cusp field of 5 to 10 percent of the center field and a mirror ratio of 2 .

\title{
INTRODUCTION
}

Magnetic fields for plasma physics experiments have been produced in many sizes, configurations, and field strengths. In many cases the experiments have been handicapped by limited power, limited field strength, or duration of experiments by insufficient power or inadequate cooling for the magnet coils. In addition, the heavy coils, support structure, and cooling passages have interfered with free access to the centerline of the magnetic fields.

Now, the rapidly developing techniques of manufacture of superconducting magnets offers a solution to these difficulties while introducing only the additional problem of liquid-helium environment for the magnetic coils. The Lewis Research Center of INASA has been studying the application of superconducing magnets to plasma physics. One such apparatus, described by Roth[1], has per- 
formed well in actual use during the past several months. This device consists of a split pair of superconducting coils each enclosed in its separate helium dewar. The separation between the dewars is adjustable by inserting support rods of different lengths. The inner diameter of the $25-\mathrm{kg}$ coils is about 7 in. and the separation can be varied from approximately 6 to 21 in. The system of coils described in this paper was designed to study the use of superconducting coils at higher fields and in other configurations than those now in use for plasma physics experiments at Lewis Research Center. Among the configurations to be studied are those deemed most suitable for stable plasma containment (such as the Ioffe cusp fields[2]). The mirror coils were designed (using the methods of refs 3 to 5 ) to produce $50 \mathrm{kG}$ on the central axis (see Fig. 1). A mirror ratio of 2 was provided and a more uniform central field was produced by inserting a third coil between the split pair. In addition a cusp field of 5 to 10 percent of the center field is produced by an array of four linear conductors ("Ioffe bars") equally spaced around the inner diameter of the coil forms. The design, fabrication, and operation of this system of superconducting coils are described.

\section{DESIGN AND FABRICATION OF COILS}

Field Requirements

The coil design to produce the 2:1 mirror ratio and maximum mirror fields of $50 \mathrm{kG}$ was straightforward. With a minimum inside diameter of $4.0 \mathrm{in}$. and using the minimum wire lengths required to produce the desired fields the coil dimensions and the calculated field were as shown in Table I and Fig. 2. 
Figure 2 shows that the design mirror ratio and field strengths are closely achieved with the coil dimensions indicated in Table I. The spacing between the coils is sufficient to provide access to the center line of the field if necessary by constructing the coil forms so that magnets can be placed in individual dewars. Additional windings on the center coil could shape the field into other desirable configurations.

The design of the cusp field, however, presented some problems of its own. It is necessary to design a system of linear conductors ("Ioffe bars") which will operate in the high field close to the inner windings of the coils. The performance of these conductors is of course affec5ed by the background field of the main coils and by the methods used to introduce the current into them.

The winding scheme of the Ioffe coils is shown in Fig. 3. Since very high current superconductors, as well as flux pumps to power them, are not available at the present time, it was necessary to design these coils of many turns of superconducting wire carying a modest current. The parameters of the coils are shown in Table I.

Calculations for field configurations such as these Ioffe bars are available in the literature (see ref. [6] for instance.), but for the present design the calculations were performed on the IBM 7090 to give tables of desired fields in terms of the geometrical dimensions of the coils. The results of this study for a quadrupole field are shown in Fig. 4(a) where the constant field strength contours are shown. Fig. $4(\mathrm{~b})$ is an iron filing pictograph which shows the magnetic lines of force produced by the Ioffe coil. 
Selection of Materials

In the design of the coils, particularly in the choice of field strengths, the availability and characteristics of superconducting materials was carefully considered. Because of the state of development of the superconducting materials and the magnitude of the desired fields, it was decided to use $\mathrm{No}-\mathrm{Zr}$, NoTi, or a combination of the two for these coils. An evaluation of available NbZr and NbTi materials was undertaken to determine the best material for winding the coils. Single wires 0.010 in. in diameter and 7 -stranded cable of the same wire were procured and evaluated. The results of the evaluation were that NB-25\% Zr in the 7-stranded cable would produce the desired coils most economically considering cost of material and time required to fabricate the coils.

The quality of the material as a superconductor varied widely depending on the wire manufacturer, the copper plate on the wire, the presence of indium as a potting metal for the cables, and the insulation. If the copper plating was thin and flaky, the cable or wire was much poorer magnet material and more subject to damage when the coils were ariven normal. The thickness and uniformity of the insulation was also an important consideration in eliminating shorts between turns.

The materials selected as a result of these preliminary tests was more than adequate to produce the design coils and has performed well in use. Description of Coils

The actual coils and forms produced are shown in Fig. 5. The mirror coils are wound of 7 -strand $\mathrm{Nb}-25 \% \mathrm{Zr}$ cable, each wire of which is individually copperplated. The entire cable, after stranding, is impregnated with pure indium metal (99.999\% indium) and insulated with mylar insulation. 
There have been no significant differences in performance between the design coils and the fabricated coils. The production cable is actually somewhat smaller in diameter than that used in the design. This difference did not complicate the winding of the coils. Aluminum foil was wound between the layers to help provide protection for the coils on going normal.

The center coil (Coil B) is wound of 7-strand cable also. The cable is identical to that of coils $A$ and $C$ except it is not impregnated with indium metal.

Coil D, (Ioffe bars) is wound of the same 7-strand $\mathrm{Nb}-25 \% \mathrm{Zr}$ cable as coil $\mathrm{C}$. The coil forms in all cases are stainless-steel tubing with flanges of stainless steel welded to the tubing. In the case of coil D, the turns are restrained by a half tube of stainless steel welded to a center tube of stainless steel, as shown in Fig. 3. In this coil, the superconducting cable is held in place by an epoxy potting compound which is easily removed by a solvent if changes need to be made in the number of turns or in the wire itself.

In coils A, B, and $C$, interturn insulation is provided by mylar tape but no potting compound was used. The individual turns of the coils are wound tightly under sufficient tension to prevent movement of the conductors in the magnetic field. Such movement almost invariably causes a transition from the superconducting to normal resistance. The turns are further restrained by a winding of nylon fishing line on the outside of the wire.

The normal-to-superconducting contacts were formed by soldering with indium solder, the superconducting cables to copper channels that were bolted to the coil forms using nylon bolts for insulation. The copper channels were soldered to mesh copper conductors that could be fanned out to insure good contact with the liquid helium. The resistance of the connections (superconducting to normal) 
made in this manner were of the order of $10^{-7}$ ohms and were quite sufficient for testing the magnet coils. No persistent switches were included in the prototype coils, but such switches could easily be included if desired.

RESULTS AND DISCUSSION

The results of the preliminary tests on the separate coils and the tests on the final configuration are given in a series of graphs. Figures 6 to 10 show the data obtained with the coils shown in Fig. 5. These figures are $X-Y$ plots of the actual tests showing the charging current on the ordinate and time on the abscissa. The curves present a charging history of the coils.

Figures 6 to 8 show the results for the mirror coils $A$ and $C$ and the center coil B tested alone with the remaining coils shorted by a silicon diode outside the dewar. The sharp break in the curve is the transition point from superconducting to normal state. It can be seen from the data that the critical currents (167, 173, and $180 \mathrm{~A}$ for coils A, C, and B respectively) are not significantly less than the design values of 175A. Since more turns were actually wound on the coils than the design requirements, the field requirements were easily met. In the case of these three coils the respective values of the magnetic field are shown on the figures also.

Figure 9 shows the result of powering the three coils $A, B$, and $C$ in series. The critical current of 152 A gave a central field on the axis of the mirror coils of $47.0 \mathrm{kG}$ which is within $3 \%$ of the design field. It is expected that final working of the superconducting-to-normal contacts and perhaps winding a few more turns on the center coil will result in operation at the design field values. 
Figure 10 shows the charging history of the Ioffe bars alone. The critical current in this run was $234 \mathrm{~A}$ which is sufficient to give the 5 to $10 \%$ of the center field of coil $B$ which is required by the design.

Tests of all four coils in series consistently resulted in transition from superconducting-to-normal operation when the current reached 85 to $95 \mathrm{~A}$. This result pointed out that series operation of all four coils is impractical and that the Ioffe bars, and perhaps the center coil, require separate power supplies. At the time of preparation of this paper these tests were underway and the results may be expected in time for the oral presentation.

The results of the tests (which show that relatively large currents are required to power coils wound with superconducting cables) indicate the need for a flux pump or some similar device for charging superconducting magnets. The need for several leads of large cross section going into the liquid-helium dewar increases the heat leak into the dewar and results in large consumption of liquid helium.

\section{CONCLUSIONS}

A superconducting magnetic bottle consisting of two mirror coils, a central field coil, and a quadrupole (Ioffe) field coil has been designed and tests of the components are described. The mirror ratio of the field is $2: 1$ and the field strength of the mirror culis is $50 \mathrm{kG}$. The finterial ajimetor of the mimror coils is 4 inches. Some degradation in current-carrying capability of the Ioffe field coil was observed due to interaction with the main magnetic field of the bottle when the coils were operated in series. 
NOTATION

a coil radius, in.

$\mathrm{B}_{0}$ field at center of coil, $\mathrm{kG}$

$\mathrm{H}$ field strength, $\mathrm{kG}$

$\mathrm{H}_{\mathrm{C}} \quad$ critical field

I current, A

I length of windings, in.

2 length of wire, ft

$\mathrm{N}_{\mathrm{L}} \quad$ number of layers

$N_{T} \quad$ number of turns

a outer diameter/inner diameter

$\beta \quad$ length of windings/inner diameter 
REFEREICES

1. Roth, J. R., Freeman, D. C., and Haid, D. A.: Superconducting Magnet Facility for Plasma Physics Research. Proc. 6th Symposium on Engr. Aspects of MHD, Pittsburgh, Pa., April 21-22, 1965.

2. Gott, Yu. V., Ioffe, M. S., and Telkovsky, V. G.: Some New Results on Confinement in Magnetic Traps. Nuclear Fusion, Supplement 2, part 3, 1962 , pp. 1045-1047.

3. Brown, Gerald V., Flax, Lawrence, Itean, Eugene C., and Laurence, James C.: Axial and Radial Magnetic Fields of Thick, Finite-Length Solenoids, NASA Tech. Rpt. TR R-170, December 1963.

4. Brown, Gerald V., and Flax, Lawrence: Superposition of Semi-Infinite Solenoids for Calculating Magnetic Fields of Thick Solenoids. Journal of Applied Physics, Vol. 35, No. 6, pp. 1764-67, June 1964.

5. Brown, Gerald V., and Flax, Lawrence: Superposition Calculation of Thick Solenoid Fields from Semi-Infinite Solenoid Tables. NASA Tech. Note TN D-2494, September 1964.

6. Uo, Koji: The Multipolar Cusp Magnetic Field and Its Application to the Adiabatic Compression of Plasma. Princeton Univ. Plasma Physics Laboratory MATT-185, April 1963. 


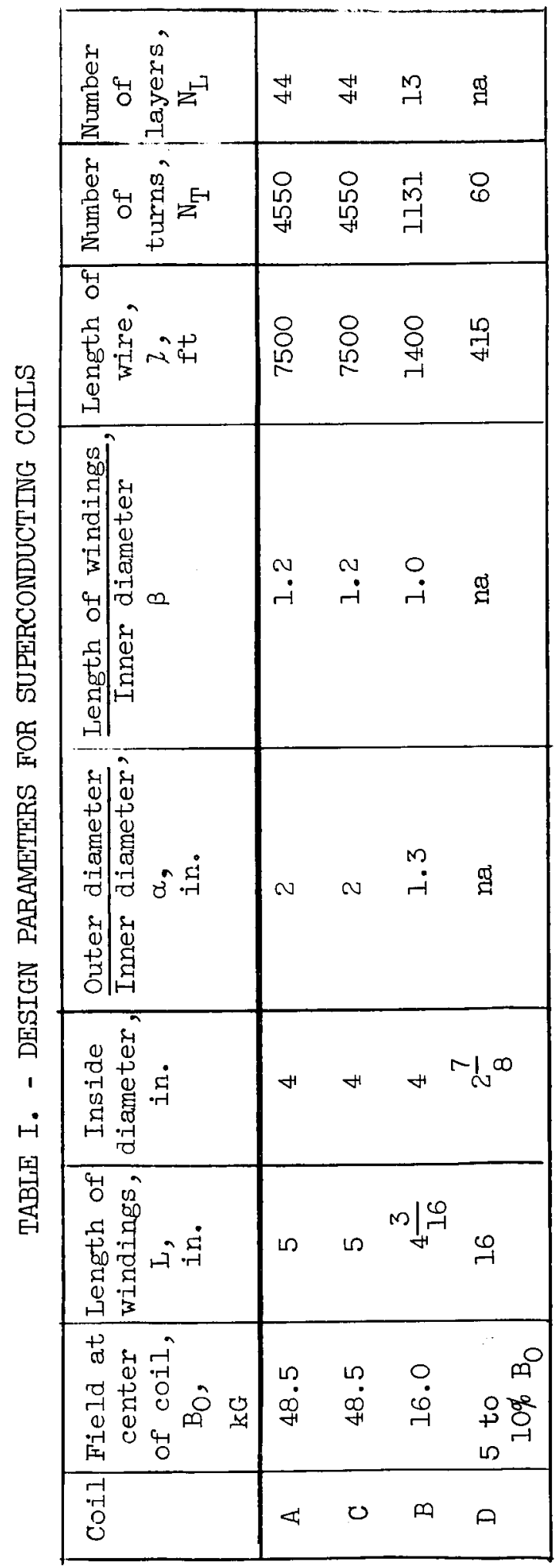




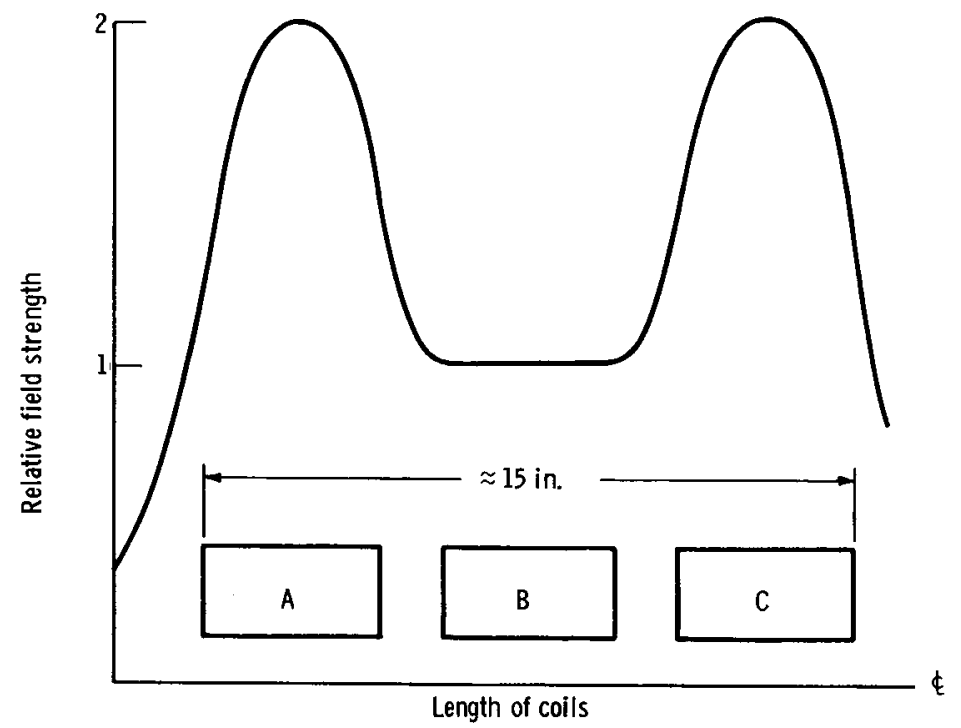

Fig. 1. Magnetic mirror configuration for plasma physics experiments.

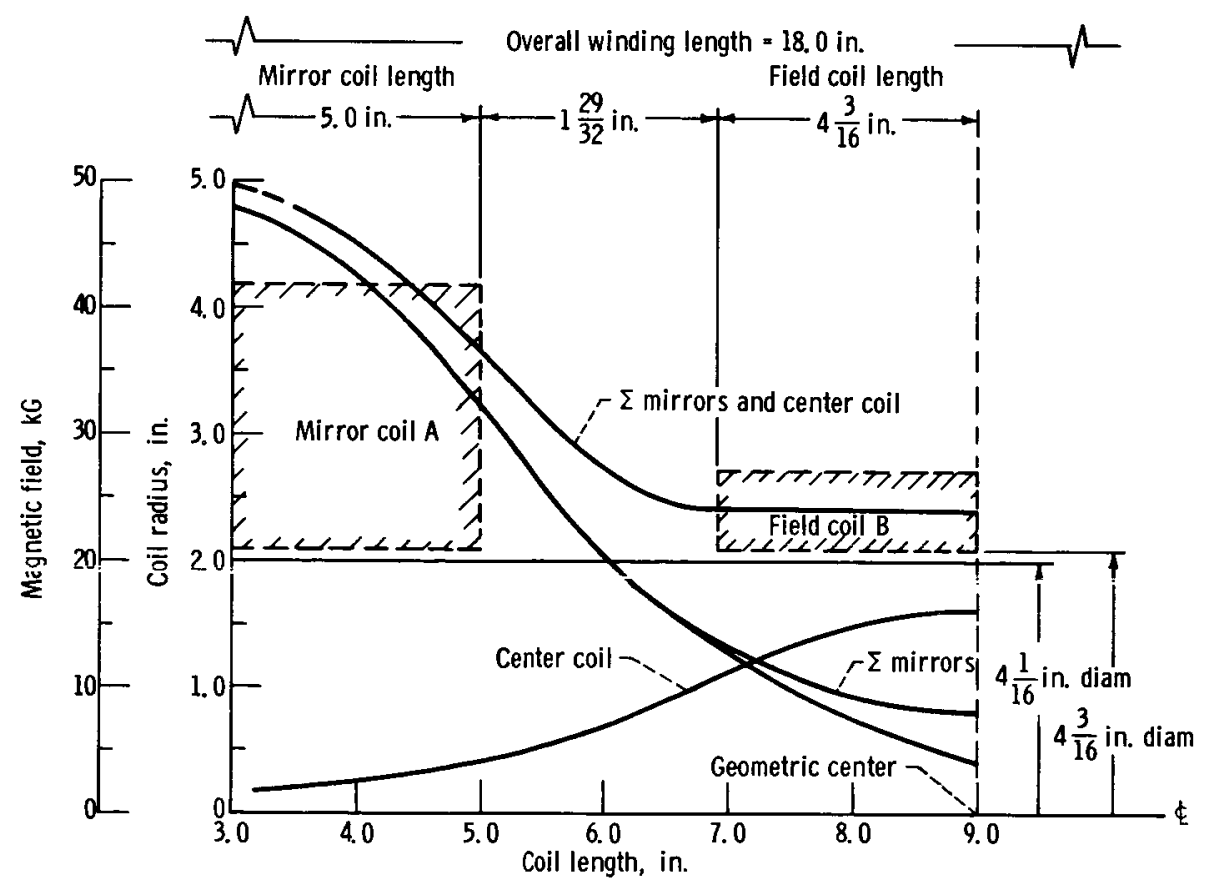

Fig. 2. Design parameters for magnetic coils (I assumed $=25 \mathrm{~A} / \mathrm{strand}$ or $175 \mathrm{~A} / 7$-strand cable, current density $=1.2 \times 10^{4} \mathrm{~A} / \mathrm{sq} \mathrm{cm}$ ). 

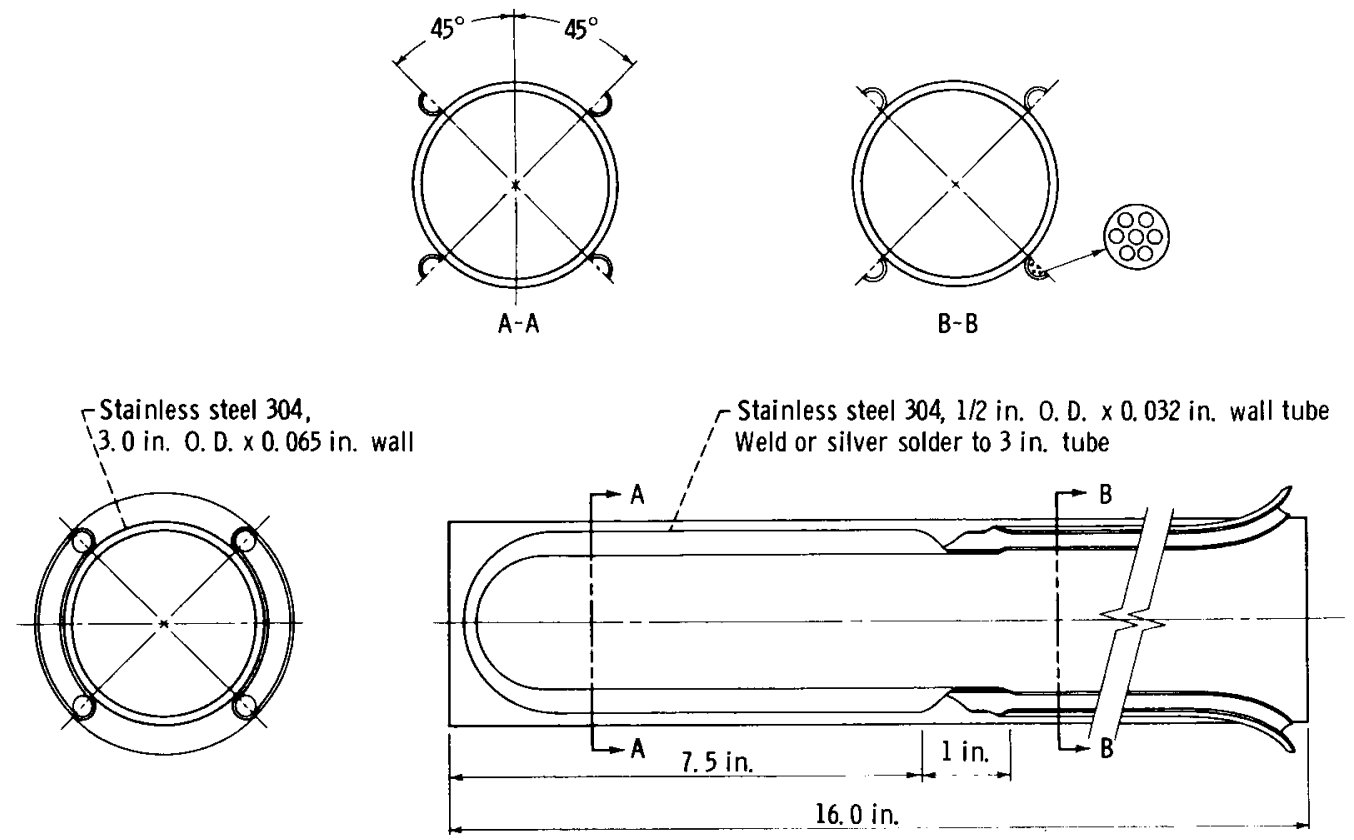

Fig. 3. loffe bar coil form.

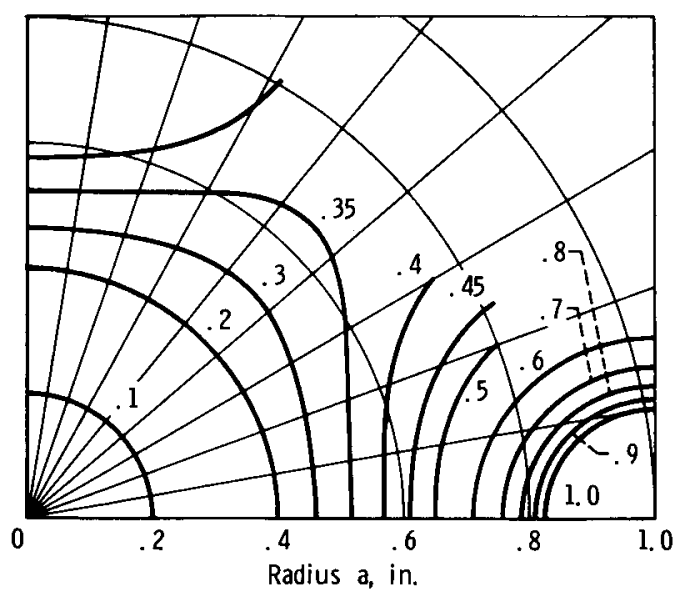

(a) Calculated cusp field. Contours of constant $\mathrm{Ha} / \mathrm{I}$.

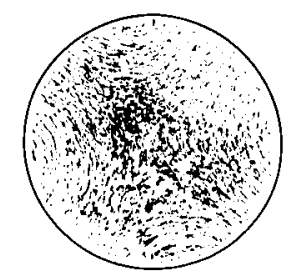

(b) Iron filing pictograph.

Fig. 4. Magnetic field of loffe bars. 
1
8
0
1
됨

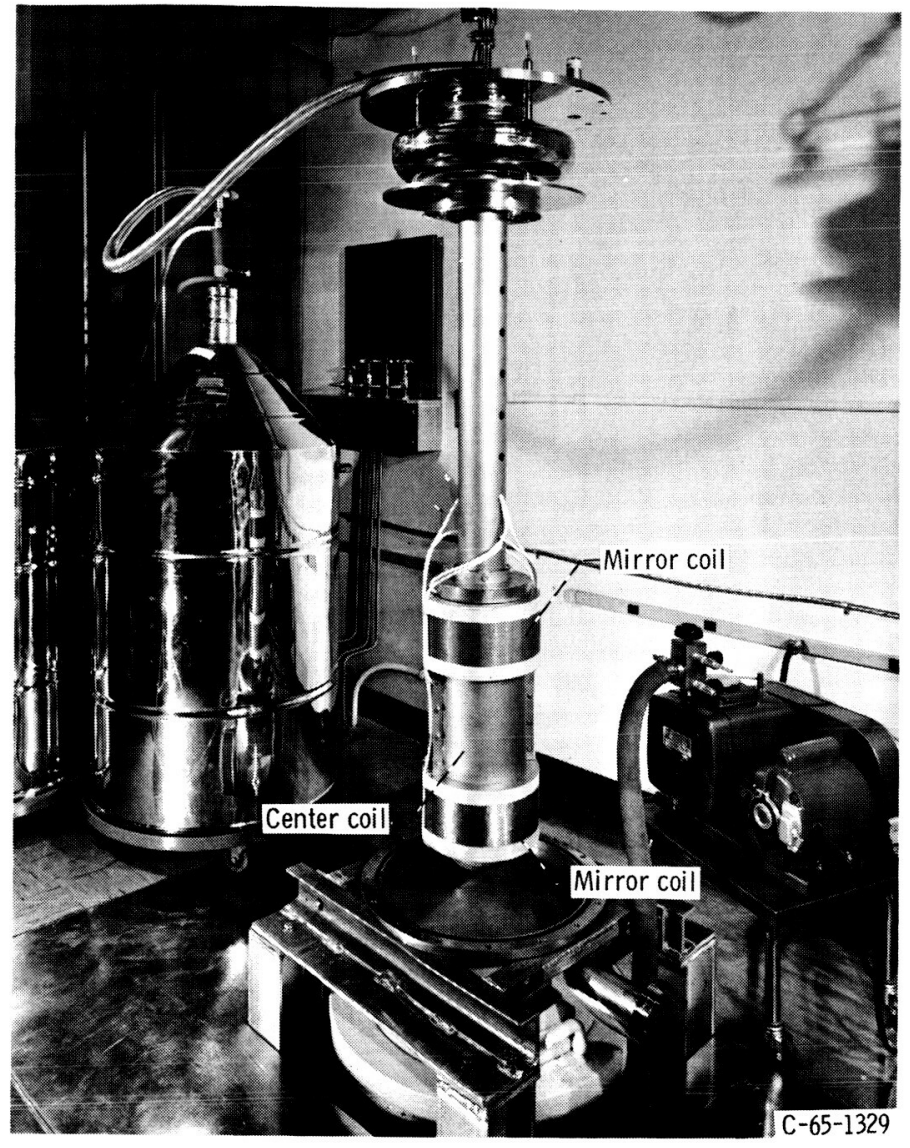

Figure 5. - Magnetic bottle.

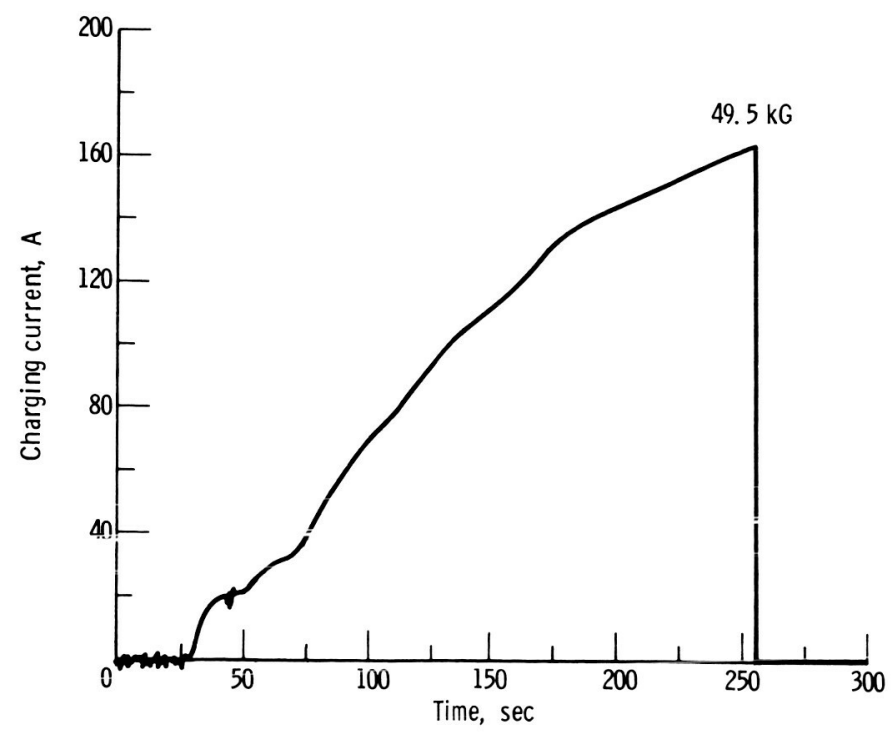

Fig. 6. Charging history, coil A alone. 


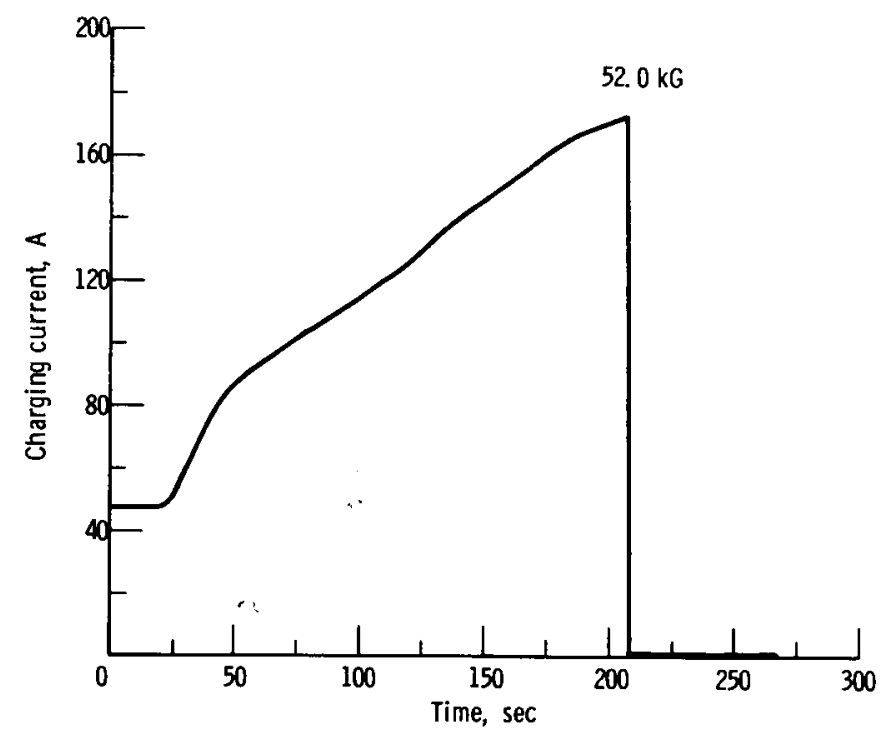

Fig. 7. Charging history, coil $\mathrm{C}$ alone.

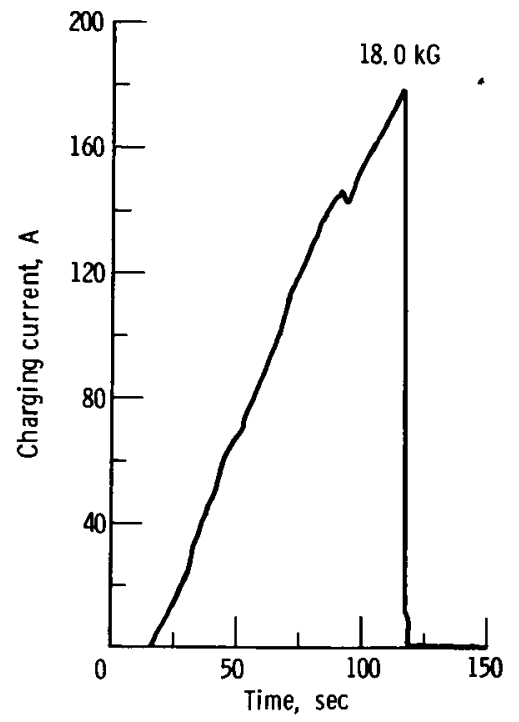

Fig. 8. Charging history, coil B alone.

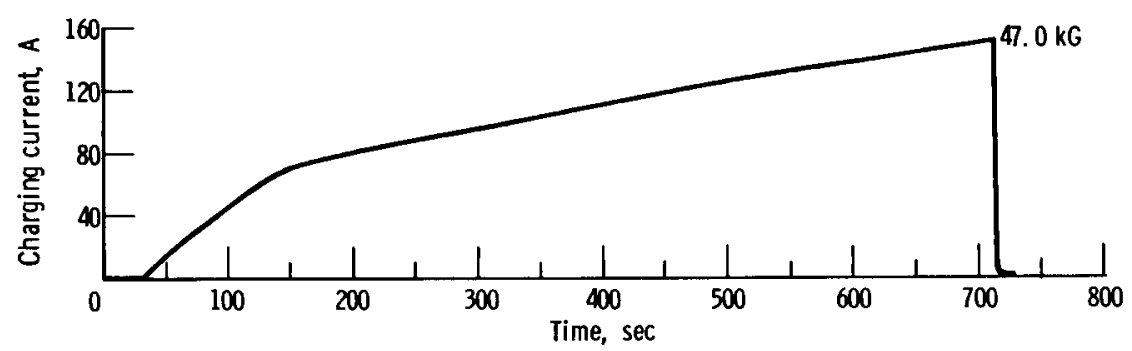

Fig. 9. Charging history, coils A, C, and B in series.

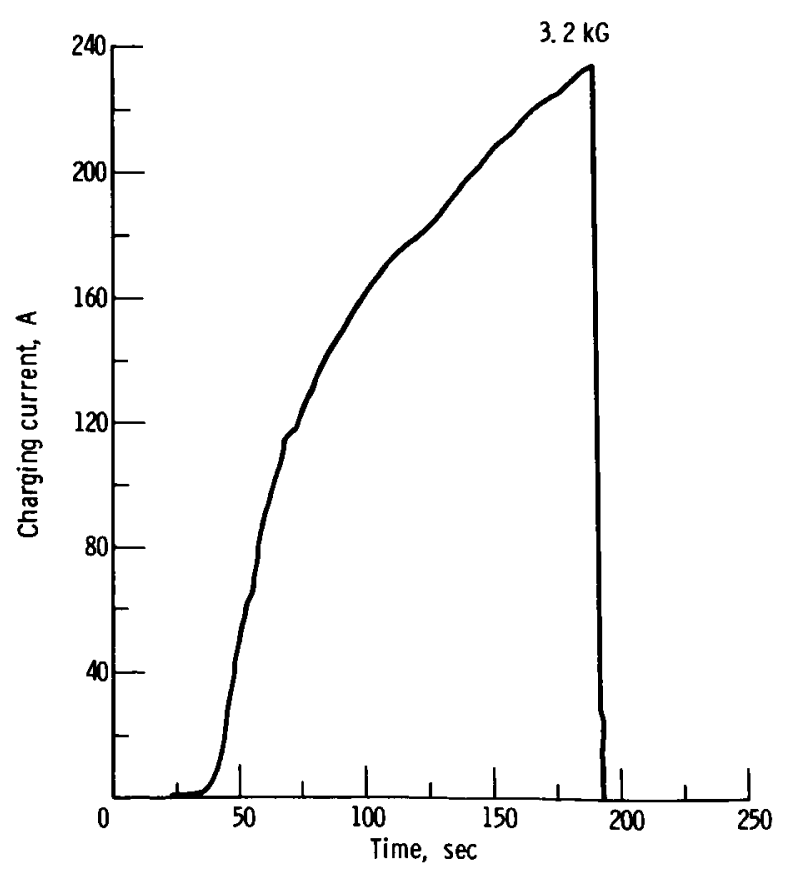

Fig. 10. Charging history, coil $D$ alone. 